\title{
FOXP2 gene and language impairment in schizophrenia: association and epigenetic studies
}

\author{
Amparo Tolosa ${ }^{1 *}$, Julio Sanjuán ${ }^{2,3}$, Adam M Dagnall ${ }^{4}$, María D Moltó ${ }^{1,3}$, Neus Herrero ${ }^{2,3}$, Rosa de Frutos ${ }^{1,3}$
}

\begin{abstract}
Background: Schizophrenia is considered a language related human specific disease. Previous studies have reported evidence of positive selection for schizophrenia-associated genes specific to the human lineage. FOXP2 shows two important features as a convincing candidate gene for schizophrenia vulnerability: FOXP2 is the first gene related to a language disorder, and it has been subject to positive selection in the human lineage.

Methods: Twenty-seven SNPS of FOXP2 were genotyped in a cohort of 293 patients with schizophrenia and 340 controls. We analyzed in particular the association with the poverty of speech and the intensity of auditory hallucinations. Potential expansion of three trinucleotide repeats of FOXP2 was also screened in a subsample. Methylation analysis of a CpG island, located in the first exon of the gene, was performed in post-mortem brain samples, as well as qRT-PCR analysis.

Results: A significant association was found between the SNP rs2253478 and the item Poverty of speech of the Manchester scale ( $p=0.038$ after Bonferroni correction). In patients, we detected higher degree of methylation in the left parahippocampus gyrus than in the right one.
\end{abstract}

Conclusions: FOXP2 might be involved in the language disorder in patients with schizophrenia. Epigenetic factors might be also implicated in the developing of this disorder.

\section{Background}

It is widely accepted that neutral drift and Darwinian positive selection have played an important role in the evolution of human features. During the last few years, research has been focused on human genome-wide scans of adaptative evolving loci to search for specific modern characteristics in this species [1]. Although most of them are related to fitness, it has been reported that some genes under positive selection in the human lineage can also confer vulnerability to some diseases [2-4].

Schizophrenia, which is considered as a disease related to the origin of Homo sapiens, could be a by-product of an adaptative process $[3,5,6]$. Previous reports have indicated a relationship between positively selected genes and schizophrenia. Crespi et al. [3] found signals of positive selection in 28 of 76 schizophrenia candidate genes that had been previously reported as positive results in association studies. Evidence of recent positive selection in the human lineage has also been found in

\footnotetext{
* Correspondence: amparo.tolosa@uv.es

'Department of Genetics, Faculty in Biology, University of Valencia, C/Doctor Moliner 50, CP:46100, Burjassot, Valencia, Spain
}

haplotypes of $M A O B$ and $G A B R B 2$ genes, which also confer an increased risk to schizophrenia [2,4]. Furthermore, brain areas that are differentially dysregulated in schizophrenia include the regions most-notably subject to differential evolutionary change along the human lineage [7-9]. In addition, it has recently been suggested that metabolic processes altered in schizophrenia evolved at a higher rate in the human lineage, when compared with the chimpanzee [10].

A selective advantage could affect the achievement of specific human capacities, such as language. In this context, TJ Crow $[9,11]$, postulates that schizophrenia is the price that Homo sapiens had to pay for the acquisition of language. Moreover, recent neuroimaging studies report impairment in brain function relevant to language processing in individuals with schizophrenia and in those who are at a genetic risk for this disease [12].

First evidence for a gene involved in language was reported in 2001, when the FOXP2 gene was identified by Lai et al. [13]. Identification of the transcriptional targets of FOXP2 revealed that this protein could regulate genes involved in development and function of the brain, genes 
under positive selection in human lineage and genes associated to schizophrenia [14]. Apart from the polyglutamine tracts, the human protein only differs in three amino acids from its ortholog in mouse, and two of these changes occurred in the human lineage after separation from the common ancestor shared with chimpanzees. Both changes are fixed in human populations, and there is evidence to support they have been under positive selection $[15,16]$.

Association studies between FOXP2 polymorphisms and susceptibility to different pathologies of language impairment, such as specific language impairment, dyslexia or autism have not produced robust results [17], but the identification of two coding mutations related to verbal dyspraxia [18]. Nevertheless there are strong evidence of the importance of the gene in development and some aspects of language [19] including the fact that CNTNAP2, a downstream target of FOXP2 has been related also to language disorders $[20,21]$. In schizophrenia, preliminary association studies have delivered controversial results [22-24]. To the best of our knowledge, no methylation study of FOXP2 has previously been done.

We hypothesized that FOXP2 could be considered a candidate gene that may confer vulnerability to schizophrenia or to the language related symptoms of this disorder. To test this hypothesis, two different analyses were carried out: 1) an association study between FOXP2 polymorphisms and schizophrenia and 2) the study of the methylation status of the FOXP2 promoter in different areas of the brain in patients and controls.

\section{Methods}

\section{Association study participants}

For the association study, 293 patients and 340 healthy unrelated controls were analyzed. All patients and controls were Caucasians of Spanish descent. Exclusion criteria included organic brain syndromes, mental retardation, severe drug abuse, or inability to understand simple questions. Participants with previous psychiatric treatment were excluded as controls.

There were no significant differences in sex or age for both groups. All patients met DSM-IV criteria for schizophrenia. The Manchester scale [25], and the psychotic symptom rating scale (PSYRATS) [26], were used respectively, to assess the clinical psychotic symptoms, with particular attention to the Poverty of speech item, and the intensity of auditory hallucinations. The mean Manchester score was $8.79(\mathrm{SD}=5.56)$ and mean PSYRATS score was $16.26(\mathrm{SD}=23.26)$. This study was approved by the local Ethics Committee. All patients signed the informed consent form.

\section{Post-mortem human brain samples}

For methylation and expression analyses, human brain samples were kindly donated by the London
Neurodegenerative Diseases Brain Bank at the Institute of Psychiatry. Grey tissue from both hemispheres of the superior temporal gyrus, parahippocampus gyrus and cingulate gyrus was obtained. For methylation analyses, one sample for each region was analyzed for both patients and controls. For expression analyses, 13 samples from patients (6 from the right hemisphere and 7 from the left hemisphere) and 12 samples from controls (9 from the right and 3 from the left hemisphere) were analyzed.

\section{Association study}

Genomic DNA was extracted from peripheral blood leukocytes by the Puregene kit (Gentra Systems, MN, USA).

A total of 27 polymorphisms were analyzed, 10 of them by polymerase chain reaction-restriction fragment length polymorphism (PCR-RFLP) and 17 by an iPLEX genotyping assay (Sequenom, CA, USA). Details of the primer sequences, PCR conditions and restriction enzymes are described in Additional files 1 and 2.

Three regions were screened for potential trinucleotide expansions: two polyQ tracts of 40 and 10 residues, located respectively in exons 5 and 6 of FOXP2, and a CGG-rich region in intron s1 close to the transcription start site. Primers flanking the three regions were designed (see Additional file 1). One primer in each pair was 5'-labeled with 6-FAM or HEX fluorophores. Fluorescent amplicons were electrophoresed with internal lane size standards in an ABI PRISM ${ }^{\circledR} 3700$ DNA Analyzer (Applied Biosystems Inc.) and length of fragments was analyzed with the GeneScan-v3.7 (Applied Biosystems, Inc.).

Statistical and genetic analyses were performed using Haploview v4.1, UNPHASED 3.10, and SSPS v13 software. Bonferroni correction was used for multiple tests. For the haplotype association study, four marker slidingwindows were used, with the exception of a five marker haplotype, for which association had been detected in a previous study [24].

\section{Methylation analysis}

DNA from brain samples was extracted using a Nucleon ${ }^{\oplus}$ Genomic DNA Extraction Kit (Tepnel Life Sciences). DNA from leukocyte samples was extracted using the Puregene kit (Gentra Systems).

DNA was fragmented with EcoRI (New England Biolabs) prior to overnight digestion with Proteinase $\mathrm{K}$ (Sigma Aldrich). DNA was cleaned, purified and concentrated using a Qiaex II kit (Qiagen). The processed DNA samples were treated with either the CpGenome ${ }^{\mathrm{mt}}$ DNA Modification Kit (Chemicon ${ }^{\oplus}$ International) or the EpiTect Bisulfite Kit (Qiagen) in accordance with the supplier's guidelines. 
DNA was amplified with specific primers for bisulphite-converted DNA (see Additional file 1). PCR fragments were cloned into the PCR 2.1 vector using the TOPO cloning kit (Invitrogen), or $\mathrm{pGEM}^{-\mathrm{T}^{\oplus}}$ vector using the pGEM-T $\mathrm{T}^{\oplus}$ Easy Vector System (Promega), and sequenced with T7 and SP6 universal primers.

\section{Expression analysis}

Total RNA was extracted with the RNeasy Lipid Tissue Mini Kit (Qiagen). Reverse transcription of $1 \mu \mathrm{g}$ of RNA was performed using SuperScriptTM III Reverse Transcriptase (Invitrogen) and random primer hexanucleotides (Promega).

Quantitative RT-PCR was performed in triplicate for each sample on an iCycler iQ Real Time PCR System (Qiagen) with Power SYBR ${ }^{\oplus}$ Green PCR Master Mix (Applied Biosystems) using a standard protocol. Specific cDNA primers for FOXP2 and RPII, used as a control gene, were designed. Sequences and PCR conditions are shown in Additional file 1. The comparative CT method $(\Delta \Delta \mathrm{CT})$ was used to measure the relative gene expression.

\section{Results}

\section{Association study}

Twenty-four intronic SNPs and three SNPs within the 5' untranslated region of FOXP2 were selected for this study. The SNPs rs13308496 and rs10254225 were monomorphic in our sample. In Figure 1, we show the location of the selected SNPs that were polymorphic in our sample. All of the SNPs were at Hardy-Weinberg equilibrium in both patients and controls, except rs717233, which deviated from HWE in controls (see Additional file 3) and was removed in further analyses. Allelic and genotypic frequencies of the twenty-four SNPs in both patients and controls are shown in Additional file 4. Minor allele frequencies for the SNPs ranged between 0.02 and 0.49 .

First, we conducted the single SNP association analysis. When all patients were included, we observed a significant association for the SNP rs10447760 at allelic frequencies, although this association did not remain after applying the conservative Bonferroni correction. When comparing patients with auditory hallucinations versus controls (see Additional file 5), significant associations were found for SNP rs2396753 and SNP rs17137124. However, after Bonferroni correction, the significant associations were also lost.

Finally, when patients with auditory hallucinations were compared with patients without hallucinations (see Additional file 6), a significant association was found for SNP rs2253478 and SNP rs1456031 in genotypic frequencies and for SNP rs2396753 in both, genotypic and allelic frequencies. However, once again, after Bonferroni correction, the significance disappeared in all cases.

Next, we carried out the haplotypic association analysis. Tests of four marker haplotypes did not provide evidence of significant associations with schizophrenia or auditory hallucinations. However, when we took into account the five marker haplotype which was found to be significant in a previous study [24], positive results were detected for the same combination of alleles: rs7803667 T/rs10447760C/rs923875A/rs2396722C/ rs2396753A ( $\chi 2=6.479 ; p=0.0109)$. Interestingly, this combination of alleles was found more frequent in controls than in schizophrenic patients with auditory hallucinations.

Linear regression was performed to evaluate the association between the SNPs and the items of the PSYRATS and Manchester scales. After Bonferroni correction was applied, only association between SNP rs2253478 and the Poverty of speech was maintained ( $\mathrm{p}$ corrected $=0.038)$.

With regard to the analyses of potential expansions of trinucleotide tracts of FOXP2, no variation was found in any of these regions in our sample. Only a single deletion of three trinucleotides at the CGG-rich region in intron s1 was identified in heterozygosis in a patient with schizophrenia.

\section{Methylation analyses}

FOXP2 has four independent transcriptional start sites that would be termed exon s1, exon 1 , exon $1 \mathrm{~b}$ and exon 2 using the conventional designations of its initiation exon. In this work, we have focused on the study of region including exon $\mathrm{s} 1$, which is considered a basal transcription start site. Figure 2 shows a diagram of the region studied.

For the region named CG1 bisulphite region, no differences were found between patients and controls or between brain areas. This region, located upstream of exon s1 was characterized by a general absence of methylation in all the samples analyzed. In the case of CG2 bisulphite region, located downstream of exon s1, a higher degree of methylation was found with respect to CG1. In addition, subtle differences were found for the parahippocampus gyrus. Within this brain area, the methylation degree is higher than in the other areas analyzed in both patients and controls. When right and left parahippocampus gyrus regions were compared, differences in the degree of methylation between patients and controls were identified (Figure 3). In patients, the degree of methylation is higher in the left hemisphere of the parahippocampus gyrus than in the right one, whereas in controls, methylation is concentrated more in the right hemisphere of the parahippocampus gyrus region. Interestingly, most of the clones that were 


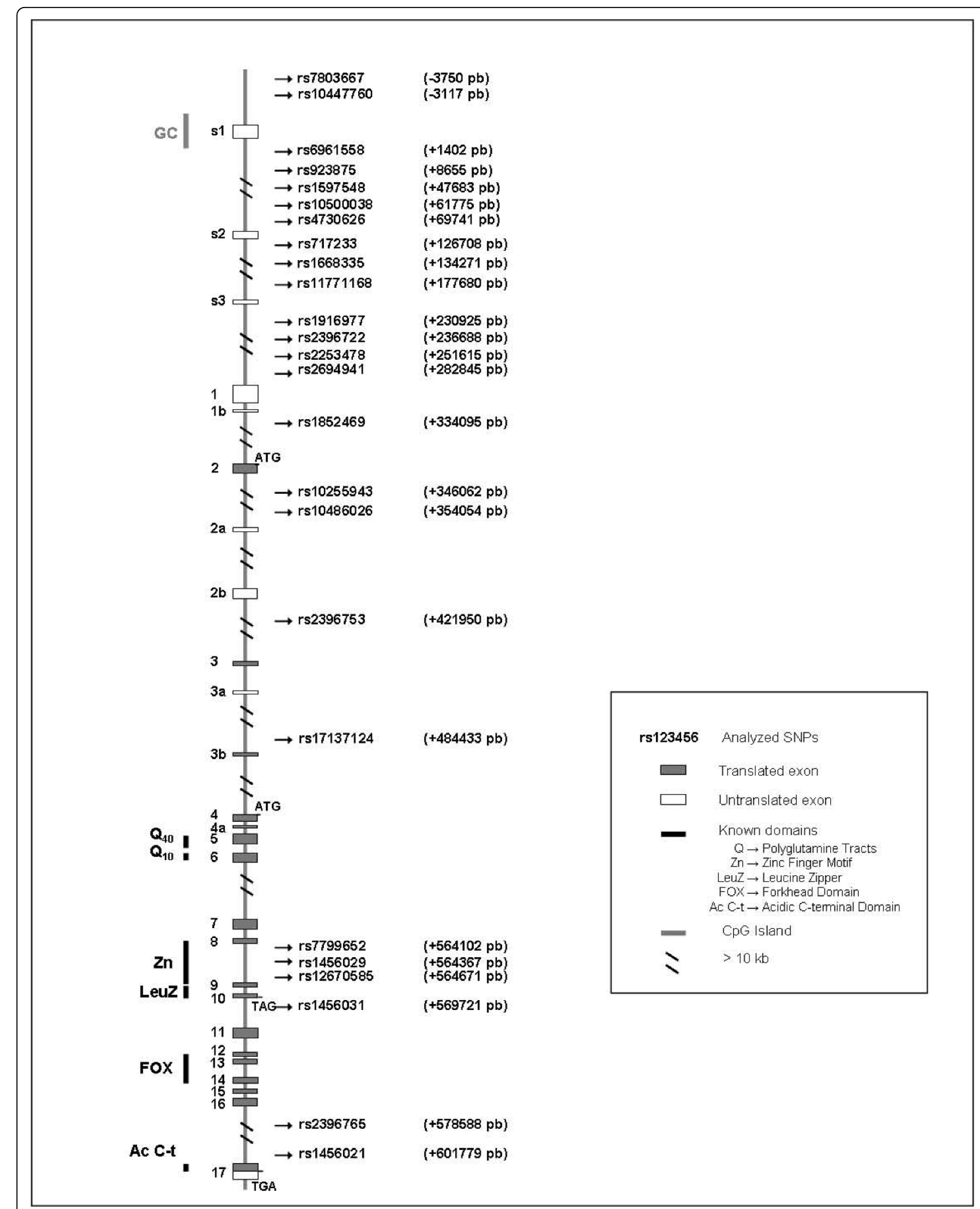

Figure 1 Structure of FOXP2 gene. Hash marks indicate introns longer than $50 \mathrm{~kb}$. Arrows indicate positions of all single nucleotide polymorphisms (SNPs) analyzed in this study: arrows indicate SNPs polymorphic in our sample. Distances of SNPs to +1 site ( $5^{\prime}$ end of s1 exon) are shown in brackets. 


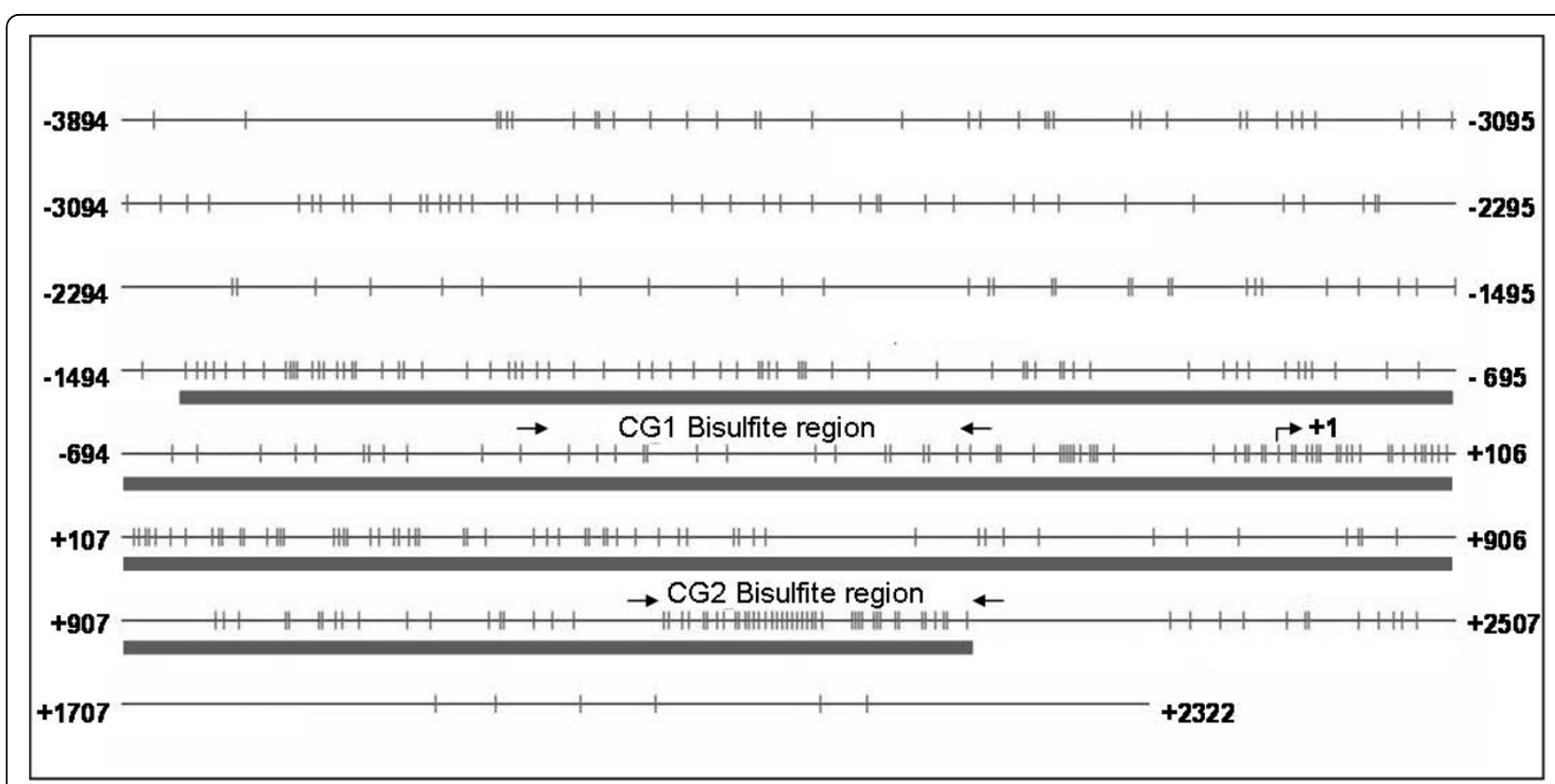

Figure 2 CpG dinucleotides in the context of the CpG island located in transcription start site. The thin black line corresponds to the sequence of DNA. Below this, a single black bar corresponds to the predicted CpG island location. Distances in base pairs to +1 site are included, as well as an arrow indicating the start site of exon s1. Primers for both regions analyzed, CG1 and CG2 are indicated with arrows.

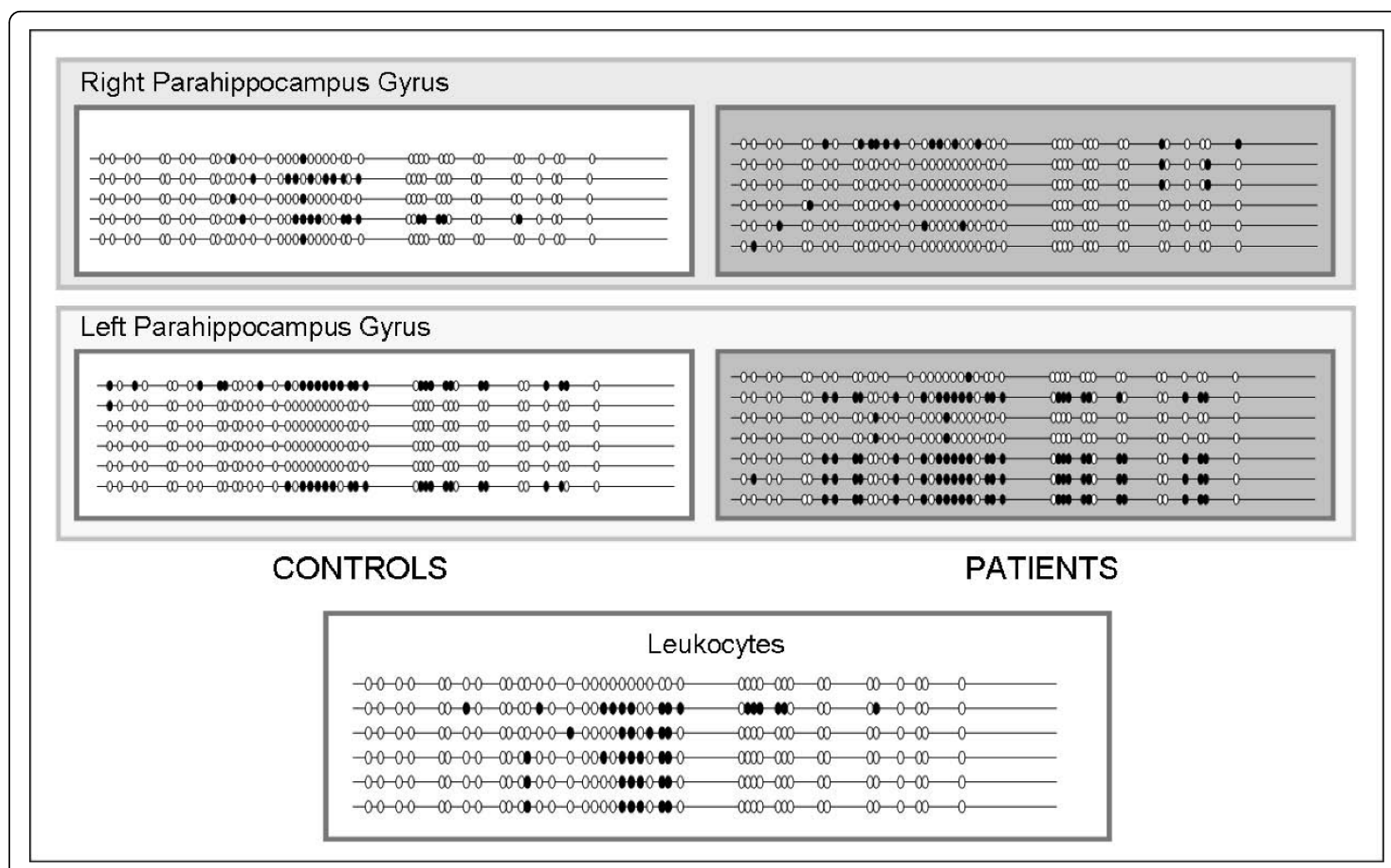

Figure 3 Bisulfite results for parahippocampus gyrus. Methylation data are represented as filled circles (methylated CpG) and empty circles (unmethylated $\mathrm{CpG}$ ) for each bacterial clone obtained. Each row of circles represents the methylation pattern based on the sequence of one cloned PCR product. 
analyzed showed similar methylation patterns (Figure 3). A high degree of methylation in the CG2 region was also found in leukocyte samples of controls. In this case, the observed methylation pattern is different from the one obtained from brain samples of controls.

Generally, a high degree of methylation on the promoter region of a gene is correlated with lower RNA expression levels. Therefore, differences in the degree of methylation could result in differential expression of the gene. When comparing the relative expression level of FOXP2 in parahippocampus gyrus between patients and controls, higher levels of FOXP2 mRNA expression were obtained in the right hemisphere in patients (Figure 4). This correlates inversely with methylation results, which show a greater degree of methylation in controls than in patients. However, when expression levels from the left and right hemispheres were compared in patients, no correlation was found, since expression is higher in left parahippocampus than in right, as well as the level of methylation. These findings do not support the idea that a high degree of methylation leads to decreased expression of the gene.

\section{Discussion}

In this study we investigated the role of FOXP2, a positively selected gene, in schizophrenia vulnerability. A SNP association study, with particular attention to language related symptoms as auditory hallucinations and poverty of speech, and a study of DNA methylation and expression of this gene were carried out.

The most important finding of this study is the significant association showed between the rs2253478 SNP and the item of Poverty of speech of the Manchester scale $(\mathrm{p}=0.038$ after Bonferroni correction). This polymorphism is located in intron $\mathrm{s} 3$, not close to any

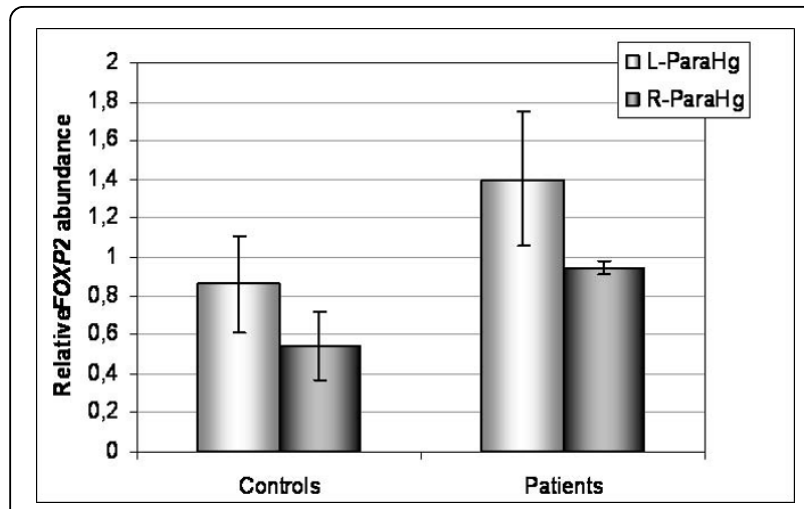

Figure 4 Levels of FOXP2 expression in parahippocampus gyrus brain area. of the promoter regions. There is no information that it could be an enhancer of splicing element. Its potential functionality has not been yet investigated, and then it is difficult to determine the biological significance of this association. Alternatively it could be in linkage disequilibrium with another polymorphism being the causative factor. In any case, our results relate the FOXP2 gene to one of the characteristic symptoms of schizophrenia, deficits in the language domain [27-29].

On the other hand, the haplotypic analysis confirmed our previous results that the $\mathrm{rs} 7803667 \mathrm{~T} / \mathrm{rs} 10447760 \mathrm{C} /$ rs923875A/rs2396722C/rs2396753A haplotype could be a protective one with respect to auditory hallucinations [24].

It has been suggested that specific language-related circuits are affected in patients with schizophrenia [12]. Therefore, it is reasonable to look for risk alleles to schizophrenia vulnerability in FOXP2, a gene for which an implication in the development of language is well accepted $[13,30]$. Nevertheless, schizophrenia is a biological entity not well defined, indicative of a phenotype too much complex for genetic analysis, which partially explains the difficulty to find the causative genetic factors. At this point, the study of language variables in order to find risk alleles in schizophrenia becomes a good alternative with respect to endophenotype approaches. Our results support this hypothesis, since significant results were found when we related language impairment in schizophrenic patients to FOXP2 polymorphisms.

In this work, we also analyzed whether the polyQ stretches at exons 5 and 6 of FOXP2 are polymorphic and if so, determine its potential association with schizophrenia vulnerability. Expansions in the number of trinucleotides repeats are frequently associated with neurodegenerative diseases [31]. However, no variation in the number of glutamines was found in our sample. This high stability is concordant with previous studies in controls, individuals with progressive movement disorders, and schizophrenic patients $[32,33]$. The role of the polyQ tracts in the FOXP2 gene is unknown. In fact, most of the members of the FOX family lack this domain. Nevertheless, the high invariability of these sequences suggests that they could be under functional constraints.

In addition to schizophrenia vulnerability due to variations in the DNA sequence, epigenetic factors regulating gene expression have also been suggested as a potential etiological mechanism in psychosis [34,35]. Epigenetic regulation has been increasingly associated with psychiatric disorders, with examples in depression and addiction $[36,37]$. 
In our study of DNA methylation of FOXP2 exon s1 region, we found a higher degree of methylation in the left hemisphere of the parahippocampus gyrus region in patients than in controls. From these results, we would have expected lower gene expression of FOXP2 due to repression by methylation. However, no differences were found in FOXP2 expression between controls and patients. This discrepancy could be explained by the fact that only a stretch of the CpG island, located in exon $\mathrm{s} 1$, was analyzed for methylation. The promoter region of the FOXP2 gene has not been well defined, and regulation of the gene is more complex than was initially thought (non published personal data, [38]). The finding that expression data show a trend of more expression in patients than in controls would indicate that a decrease of neural processes controlled by the protein FOXP2, a repressor of transcription, is produced in patients. Hippocampal and parahippocampal volume reduction is one of the most consistent findings in schizophrenia [39]. Moreover, in a meta-analysis of brain volumes in relatives of patients with schizophrenia, hippocampal reduction was the largest difference between relatives and healthy controls [40]. These findings suggest hippocampal volume as a potential end of phenotype for genetic studies in schizophrenia.

Our study has some limitations. First, the language skills evaluated in this work include only two items of the Manchester scale. Since the strongest result is related to one of these items, we would recommend a systematic exploration of language variables in schizophrenic patients. Therefore, it would be valuable to explore different aspects of language in future studies. Second, we have used a small sample in the methylation and expression analyses so further studies with a larger sample would be necessary in order to confirm our preliminary results. Finally, other variables which could affect methylation, such as medication or age, should be considered. In spite of these limitations, this study suggests the use of the language related disorder as alternative phenotypes in schizophrenia for genetic studies. On the other hand, although the results are not conclusive, this is the first epigenetic study of FOXP2 in schizophrenia, opening a new way in which this gene could be related to this disorder.

\section{Conclusions}

Our results do not support the involvement of FOXP2 in the vulnerability to schizophrenia as a global syndrome. Nevertheless, this gene might be implicated in schizophrenia through its role in language impairment. Epigenetic mechanisms affecting the expression of FOXP2 might contribute to the development of schizophrenia and related neurodevelopmental disorders.

\section{Additional material}

\begin{tabular}{|c|}
\hline $\begin{array}{l}\text { Additional file 1: Sequences of primers used in the association } \\
\text { study, analysis of potential expansions of trinucleotides, } \\
\text { methylation analyses and quantitative PCR. For SNP rs6961558, one } \\
\text { of the primers was modified in order to create a restriction enzyme } \\
\text { target depending on the allele in the sequence. Modified nucleotide is } \\
\text { shown in grey. }\end{array}$ \\
\hline Additional file 2: RFLPs conditions. \\
\hline $\begin{array}{l}\text { Additional file } 3 \text { : Results for Hardy-Weinberg equilibrium test in } \\
\text { patients and controls. }\end{array}$ \\
\hline $\begin{array}{l}\text { Additional file 4: Genotype and allele frequencies of the analyzed } \\
\text { SNPs in patients and controls. }{ }^{a} \text { tests in which expected values for } \\
\text { more than one class are lower than } 5 .{ }^{*} \text { it corresponds to corrected } \\
p \text { value (Bonferroni correction). }\end{array}$ \\
\hline $\begin{array}{l}\text { Additional file 5: Genotype and allele frequencies of the analyzed } \\
\text { SNPs in patients with auditory hallucinations (AH) and controls. }{ }^{\text {a }} \\
\text { tests in which expected values for more than one class are lower than } 5 \text {. } \\
\text { b tests in which due to lack of some classes, it was used a table } 2 \times 2 \\
\text { instead a } 3 \times 2 .{ }^{*} \text { it corresponds to corrected p value (Bonferroni } \\
\text { correction). }\end{array}$ \\
\hline $\begin{array}{l}\text { Additional file 6: Genotype and allele frequencies of the analyzed } \\
\text { SNPs in patients with auditory hallucinations (AH) and patients } \\
\text { without } \mathrm{AH} \text {. }^{\text {a }} \text { tests in which expected values for more than one class } \\
\text { are lower than } 5 \text {. }^{\text {b }} \text { tests in which expected values for one class are lower } \\
\text { than } 2 \text {. }^{c} \text { tests in which due to lack of some classes, it was used a table } \\
2 \times 2 \text { instead a } 3 \times 2 .^{*} \text { it corresponds to corrected p value (Bonferroni } \\
\text { correction). }\end{array}$ \\
\hline
\end{tabular}

Acknowledgements

This work was supported by ISCIII, PI05/2332, and CIBERSAM.

\section{Author details}

${ }^{1}$ Department of Genetics, Faculty in Biology, University of Valencia, C/Doctor Moliner 50, CP:46100, Burjassot, Valencia, Spain. ${ }^{2}$ Psychiatric Unit, Faculty in Medicine, University of Valencia, Avda Blasco Ibáñez 15, CP46010, Valencia, Spain. ${ }^{3}$ CIBERSAM, ISCIII, Avda Blasco Ibáñez 15, CP:46010, Valencia, Spain. ${ }^{4}$ SANE POWIC, Warnerford Hospital, OX3 7JX, Oxford, UK.

\section{Authors' contributions}

AT participated in the experimental procedure, analysis of results and the draft of the manuscript. AMD participated in the experimental procedure and the draft of the manuscript. MDM, and JS participated in the conception and the design of the study. RF conceived of the study, and participated in its design and coordination and helped to draft the manuscript. All authors contributed to and have approved the final manuscript.

\section{Competing interests}

The authors declare that they have no competing interests.

Received: 7 February 2010 Accepted: 22 July 2010

Published: 22 July 2010

\section{References}

1. Sabeti PC, Varilly P, Fry B, Lohmueller J, Hostetter E, Cotsapas C, Xie X, Byrne EH, McCarroll SA, Gaudet R, et al: Genome-wide detection and characterization of positive selection in human populations. Nature 2007, 449:913-918.

2. Carrera N, Sanjuan J, Molto MD, Carracedo A, Costas J: Recent adaptive selection at MAOB and ancestral susceptibility to schizophrenia. Am J Med Genet B Neuropsychiatr Genet 2009, 150B:369-374.

3. Crespi B, Summers K, Dorus S: Adaptive evolution of genes underlying schizophrenia. Proc Biol Sci 2007, 274:2801-2810. 
4. Lo WS, Xu Z, Yu Z, Pun FW, Ng SK, Chen J, Tong KL, Zhao C, Xu X, Tsang SY, et al: Positive selection within the Schizophrenia-associated GABA(A) receptor beta2 gene. PLoS ONE 2007, 2:e462.

5. Dean $B$ : Is schizophrenia the price of human central nervous system complexity? Aust N Z J Psychiatry 2009, 43:13-24

6. Pearlson GD, Folley BS: Schizophrenia, psychiatric genetics, and Darwinian psychiatry: an evolutionary framework. Schizophr Bull 2008, 34:722-733.

7. Brune M: Schizophrenia-an evolutionary enigma? Neurosci Biobehav Rev 2004, 28:41-53.

8. Burns J: The social brain hypothesis of schizophrenia. World Psychiatry 2006, 5:77-81

9. Crow TJ: Schizophrenia as the price that homo sapiens pays for language: a resolution of the central paradox in the origin of the species. Brain Res Brain Res Rev 2000, 31:118-129.

10. Khaitovich P, Lockstone HE, Wayland MT, Tsang TM, Jayatilaka SD, Guo AJ, Zhou J, Somel M, Harris LW, Holmes E, et al: Metabolic changes in schizophrenia and human brain evolution. Genome Biol 2008, 9:R124.

11. Crow TJ: The 'big bang' theory of the origin of psychosis and the faculty of language. Schizophr Res 2008, 102:31-52.

12. Li $X$, Branch CA, Delisi LE: Language pathway abnormalities in schizophrenia: a review of fMRI and other imaging studies. Curr Opin Psychiatry 2009, 22:131-139.

13. Lai CS, Fisher SE, Hurst JA, Vargha-Khadem F, Monaco AP: A forkheaddomain gene is mutated in a severe speech and language disorder. Nature 2001, 413:519-523.

14. Vernes SC, Spiteri E, Nicod J, Groszer M, Taylor JM, Davies KE, Geschwind DH, Fisher SE: High-throughput analysis of promoter occupancy reveals direct neural targets of FOXP2, a gene mutated in speech and language disorders. Am J Hum Genet 2007, 81:1232-1250.

15. Enard W, Przeworski M, Fisher SE, Lai CS, Wiebe V, Kitano T, Monaco AP, Paabo S: Molecular evolution of FOXP2, a gene involved in speech and language. Nature 2002, 418:869-872.

16. Zhang J, Webb DM, Podlaha O: Accelerated protein evolution and origins of human-specific features: Foxp2 as an example. Genetics 2002, 162:1825-1835.

17. Fisher SE: Tangled webs: tracing the connections between genes and cognition. Cognition 2006, 101:270-297.

18. MacDermot KD, Bonora E, Sykes N, Coupe AM, Lai CS, Vernes SC, VarghaKhadem F, McKenzie F, Smith RL, Monaco AP, et al: Identification of FOXP2 truncation as a novel cause of developmental speech and language deficits. Am J Hum Genet 2005, 76:1074-1080.

19. Newbury DF, Fisher SE, Monaco AP: Recent advances in the genetics of language impairment. Genome Med 2010, 2:6.

20. Vernes SC, Newbury DF, Abrahams BS, Winchester L, Nicod J, Groszer M, Alarcón M, Oliver PL, Davies KE, Geschwind DH, et al: A functional genetic link between distinct developmental language disorders. $N$ Engl J Med 2008, 359:2337-2345.

21. Poot M, Beyer V, Schwaab I, Damatova N, Van't Slot R, Prothero J, Holder SE, Haaf T: Disruption of CNTNAP2 and additional structural genome changes in a boy with speech delay and autism spectrum disorder. Neurogenetics 2010, 11:81-89.

22. Jung SM, Jung BJ, Cho JS, Park JM: FOXP2 gene possibly associated with Korean schizophrenic patients. European Neuropsychopharmacology. The Journal of the European College of Neuropsychopharmacology 2008, 18: S4.

23. Sanjuan J, Tolosa A, Gonzalez JC, Aguilar EJ, Molto MD, Najera C, de Frutos R: FOXP2 polymorphisms in patients with schizophrenia. Schizophr Res 2005, 73:253-256.

24. Sanjuan J, Tolosa A, Gonzalez JC, Aguilar EJ, Perez-Tur J, Najera C, Molto MD, de Frutos R: Association between FOXP2 polymorphisms and schizophrenia with auditory hallucinations. Psychiatr Genet 2006, 16:67-72.

25. Krawiecka M, Goldberg D, Vaughan M: A standardized psychiatric assessment scale for rating chronic psychotic patients. Acta Psychiatr Scand 1977, 55:299-308.

26. Haddock G, McCarron J, Tarrier N, Faragher EB: Scales to measure dimensions of hallucinations and delusions: the psychotic symptom rating scales (PSYRATS). Psychol Med 1999, 29:879-889.

27. Covington MA, He C, Brown C, Naci L, McClain JT, Fjordbak BS, Semple J, Brown J: Schizophrenia and the structure of language: the linguist's view. Schizophr Res 2005, 77:85-98.
28. Delisi LE: Speech disorder in schizophrenia: review of the literature and exploration of its relation to the uniquely human capacity for language. Schizophr Bull 2001, 27:481-496.

29. McKenna PJ, Oh T: Schizophrenic speech Cambridge University Press 2005.

30. Watkins KE, Dronkers NF, Vargha-Khadem F: Behavioural analysis of an inherited speech and language disorder: comparison with acquired aphasia. Brain 2002, 125:452-464.

31. Margolis RL, Abraham MR, Gatchell SB, Li SH, Kidwai AS, Breschel TS, Stine OC, Callahan C, McInnis MG, Ross CA: cDNAs with long CAG trinucleotide repeats from human brain. Hum Genet 1997, 100:114-122.

32. Bruce HA, Margolis RL: FOXP2: novel exons, splice variants, and CAG repeat length stability. Hum Genet 2002, 111:136-144.

33. Laroche F, Ramoz N, Leroy S, Fortin C, Rousselot-Paillet B, Philippe A, Colleaux L, Bresson JL, Mogenet A, Golse B, et al: Polymorphisms of coding trinucleotide repeats of homeogenes in neurodevelopmental psychiatric disorders. Psychiatr Genet 2008, 18:295-301.

34. Kato C, Petronis A, Okazaki Y, Tochigi M, Umekage T, Sasaki T: Molecular genetic studies of schizophrenia: challenges and insights. Neurosci Res 2002, 43:295-304.

35. Petronis $A$ : The origin of schizophrenia: genetic thesis, epigenetic antithesis, and resolving synthesis. Biol Psychiatry 2004, 55:965-970.

36. Mill J, Tang T, Kaminsky Z, Khare T, Yazdanpanah S, Bouchard L, Jia P, Assadzadeh A, Flanagan J, Schumacher A, et al: Epigenomic profiling reveals DNA-methylation changes associated with major psychosis. Am J Hum Genet 2008, 82:696-711.

37. Tsankova N, Renthal W, Kumar A, Nestler EJ: Epigenetic regulation in psychiatric disorders. Nat Rev Neurosci 2007, 8:355-367.

38. Schroeder DI, Myers RM: Multiple transcription start sites for FOXP2 with varying cellular specificities. Gene 2008, 413:42-48.

39. Vita A, De Peri L, Silenzi C, Dieci M: Brain morphology in first-episode schizophrenia: a meta-analysis of quantitative magnetic resonance imaging studies. Schizophr Res 2006, 82:75-88.

40. Boos HB, Aleman A, Cahn W, Hulshoff PH, Kahn RS: Brain volumes in relatives of patients with schizophrenia: a meta-analysis. Arch Gen Psychiatry 2007, 64:297-304.

\section{Pre-publication history}

The pre-publication history for this paper can be accessed here: http://www.biomedcentral.com/1471-2350/11/114/prepub

\section{doi:10.1186/1471-2350-11-114}

Cite this article as: Tolosa et al:: FOXP2 gene and language impairment in schizophrenia: association and epigenetic studies. BMC Medical Genetics 2010 11:114

\section{Submit your next manuscript to BioMed Central and take full advantage of:}

- Convenient online submission

- Thorough peer review

- No space constraints or color figure charges

- Immediate publication on acceptance

- Inclusion in PubMed, CAS, Scopus and Google Scholar

- Research which is freely available for redistribution

Submit your manuscript at www.biomedcentral.com/submit
C Biomed Central 